\title{
Role of Lysophosphatidylcholine in the Inhibition of Endothelial Cell Motility by Oxidized Low Density Lipoprotein
}

\author{
Gurunathan Murugesan and Paul L. Fox \\ Department of Cell Biology, Cleveland Clinic Research Institute, Cleveland, Ohio 44195
}

\begin{abstract}
Endothelial cell (EC) movement is required for the development and repair of blood vessels. We have previously shown that LDL oxidized by transition metals almost completely suppressed the wound-healing migratory response of vascular EC in vitro. We now report that lysophosphatidylcholine (lysoPC), a lipid component of oxidized LDL, has an important role in the antimigratory activity of the lipoprotein. Purified 1-palmitoyl lysoPC inhibited movement with a halfmaximal activity at $\mathbf{1 2 - 1 5} \mu \mathrm{M}$, and near complete inhibition at $20 \mu \mathrm{M}$; the inhibitory concentration of lysoPC was consistent with its abundance in oxidized LDL. The inhibition was not due to cytotoxicity since protein synthesis was unaffected and since EC movement was restored after removal of lysoPC. Lysophospholipid activity was dependent on lipid structure. LysoPC's containing 1-position $\mathrm{C}_{16}$ or $\mathrm{C}_{18}$ saturated fatty acids were antimigratory, but those containing $\mathrm{C}_{\leq 14}$ saturated fatty acids or polyunsaturated fatty acids were not. The activity of 1-palmitoyl lysolipids with various head groups was examined. Lysophosphatidylinositol was more antimigratory than lysophosphatidylglycerol and lysophosphatidylcholine, which were more potent than lysophosphatidylserine and lysophosphatidylethanolamine. Monoglyceride was inactive while lysophosphatidate had promigratory activity. These results are consistent with head group size rather than charge as a critical determinant of activity. To show that lysophospholipids within an intact lipoprotein were active, LDL was treated with bee venom phospholipase $\mathrm{A}_{2}\left(\mathrm{PLA}_{2}\right)$. The modified lipoprotein inhibited EC movement to the same extent as iron-oxidized LDL and antimigratory activity correlated with the amount of lysoPC formed. To determine antimigratory activity of lysoPC present in oxidized LDL, lipid extracts from oxidized LDL were fractionated by normal phase HPLC. The fraction comigrating with lysoPC had nearly the same activity as the total extract confirming that lysoPC (or a co-eluting lipid) was a major antimigratory molecule in oxidized LDL. These studies demonstrate that lysoPC in oxidized LDL limit EC wound healing responses in vitro, and suggest a possible role for lysolipids in limiting endothelial regeneration after
\end{abstract}

A portion of this work has appeared in abstract form (Murugesan, G., and P.L. Fox. 1994. Circulation. 90:I-354).

Address correspondence to Dr. Paul L. Fox, Department of Cell Biology, Cleveland Clinic Research Institute, NC10, 9500 Euclid Avenue, Cleveland, OH 44195. Phone: 216-444-8053; FAX: 216-4449404.

Received for publication 12 October 1995 and accepted in revised form 28 March 1996.

J. Clin. Invest.

(C) The American Society for Clinical Investigation, Inc.

0021-9738/96/06/2736/09 \$2.00

Volume 97, Number 12, June 1996, 2736-2744 a denuding injury in vivo. (J. Clin. Invest. 1996. 97:27362744.) Key words: atherosclerosis • cell migration • endothelial cells • lysophospholipids • oxidized low density lipoprotein

\section{Introduction}

Endothelial cells $(\mathrm{EC})^{1}$ form a continuous monolayer at the lumenal surface of all blood vessels and are vital for the maintenance and control of vessel wall function. The integrity of the endothelium, however, may be challenged by normal EC turnover, or by traumatic injury after balloon angioplasty, vascular grafting, organ implantation, or other pathological damage. Reendothelialization of injured vessels is a critical event during the restoration of the vessel wall homeostasis. While both migration and proliferation of EC from adjacent regions can occur, the former appears to be the initiating and rate-limiting event as it releases EC from contact inhibition and leads to subsequent cell proliferation $(1,2)$. The same EC processes are involved in the formation of new capillaries during development and during tumor angiogenesis (3).

EC migration is highly regulatable in vitro. Promigratory agents include tumor-derived factors (4), tumor-promoting phorbol esters (5), vascular endothelial growth factor (6), scatter factor (7), chemokines (8), and acidic (9) and basic (10) fibroblast growth factors. Although most agonists similarly affect the movement of micro- and macrovascular EC, differential responses of these cells have been reported (11). Plasma lipoproteins are in continuous contact with vascular endothelium and as such are candidates for physiological regulators of endothelial functions such as migration and proliferation. Consistent with this concept, both LDL (12) and high density lipoprotein (13) have been shown to increase reendothelialization of in vitro wounds by stimulating EC proliferation. Recently, we have shown that high density lipoprotein, and to a lesser extent LDL, directly stimulates EC migration by a signal transduction pathway that is distinct from fibroblast growth factor (14).

Compared with the extensive list of known agonists, far less is known about inhibitors of EC movement. Protein inhibitors of aortic EC movement include transforming growth factor $\beta 1$, fibronectin, and leukemia inhibitory factor $(15,16)$. We and others have shown that LDL that has been oxidatively modified by incubation with iron or copper ions, but not unmodified LDL, suppresses EC movement in vitro (17, 18). Metal ion-oxidized LDL is characterized by altered chemical and physical properties including elevated levels of cholesterol, a diverse array of oxidized sterols and fatty acids, lysophosphatidylcholine (lysoPC), degradation fragments of apo B-100, and

1. Abbreviations used in this paper: $\mathrm{EC}$, endothelial cell; lysoPA, lysophosphatidic acid; lysoPC, lysophosphatidylcholine; lysoPE, lysophosphatidylethanolamine; lysoPG, lysophosphatidylglycerol; lysoPI, lysophosphatidylinositol; lysoPS, lysophosphatidylserine; PC, phosphatidylcholine; $\mathrm{PLA}_{2}$, phospholipase $\mathrm{A}_{2}$; TBA, thiobarbituric acid. 
increased flotation density and electrophoretic mobility (19). In addition to inducing foam cell formation in cultured macrophages (20), oxidized LDL has multiple properties that may contribute to the atherogenic process, e.g., it is chemotactic for blood monocytes (21), T-lymphocytes (22), and smooth muscle cells (23), and is cytotoxic to cultured cells including SMC (24) (see references 25 and 26 for reviews). Furthermore, recent biochemical and immunological studies indicate that oxidized LDL is present in atherosclerotic lesions (25), and may in fact be present in plasma (27). The evidence that lipid oxidation has a causative role in lesion formation is largely circumstantial and based on in vitro studies. However, recent data shows that antioxidants retard lesion formation in animal models (28), and some may be associated with reduced risk of cardiovascular disease in humans (29).

Several lipid constituents in oxidized LDL have been shown to be responsible for the cellular activities of the intact particle. $7 \beta$-hydroperoxycholestenol is the primary cytotoxin of oxidized LDL (30) and 4-hydroxynonenal activates EC phospholipase D (31). Of the known lipid components of oxidized LDL, lysophosphatidylcholine (lysoPC) is perhaps the most pluripotent (see Discussion). In our previous report we showed that at least half of the antimigratory activity of oxidized LDL was recovered in the lipid-containing solvent extract (17). We now show that lysoPC, a major lipid constituent of oxidized LDL, is a potent antagonist of EC movement in vitro.

\section{Methods}

Materials. 1-Decanoyl, 1-lauroyl, 1-myristoyl, 1-palmitoyl, 1-stearoyl, 1-oleoyl, 1-linoleoyl, and 1-linolenoyl lysoPC, palmitoyl lysophosphatidic acid (lysoPA), dipalmitoyl phosphatidylcholine (PC), 1-monopalmitin, and 1,2-dipalmitin were obtained from Serdary Research Laboratories Inc., Canada. 1-Palmitoyl lysophosphatidylethanolamine (lysoPE), lysophosphatidylglycerol (lysoPG), lysophosphatidylinositol (lysoPI), lysophosphatidylserine (lysoPS) were from Sigma Chemical Co.(St. Louis, MO). All free fatty acids were obtained from $\mathrm{Nu}$ Chek Prep, Inc. (Elysian, MN). Solvents were from Fisher Scientific (Fair Lawn, NJ) and all other reagents from Sigma Chemical Co. Lysolipids and fatty acids were delivered to EC cultures as aqueous solutions or emulsions in phosphate-buffered saline; diacyl phospholipids, 1-monopalmitin, and 1, 2-dipalmitin were added as bath-sonicated emulsions. Fatty acids were delivered as ethanolic solutions.

Cell culture and media. EC from adult bovine aortas were isolated as described (32) and subcultured in Dulbecco's modified Eagles and Hams F12 medium (1:1; GIBCO BRL, Gaithersburg, MD) containing 5\% fetal calf serum (Hyclone Laboratories Inc., Logan, UT). EC were used between passages 5 through 20. All EC incubations were at $37^{\circ} \mathrm{C}$ in a humidified atmosphere of air containing $5 \%$ $\mathrm{CO}_{2}$. Cellular protein synthesis was measured by incorporation of $\left[{ }^{3} \mathrm{H}\right]$ leucine (New England Nuclear, Wilmington, DE) into trichloroacetic acid-precipitable material (33).

Preparation and modification of lipoproteins. LDL $(d=1.019-1.063)$ was isolated by sequential ultracentrifugation from freshly drawn, citrated normolipemic human plasma to which EDTA was added prior to ultracentrifugation (24). The purity was assessed by gel electrophoresis, and all preparations were assayed for protein (34), total cholesterol (Boehringer Mannheim Corp., Indianapolis, IN), endotoxin (Pierce Chemical Co., Rockford, IL), and thiobarbituric acid (TBA)-reactivity (35).

Oxidized LDL was prepared by dialysis against $0.9 \% \mathrm{NaCl}$ containing $5 \mu \mathrm{M} \mathrm{FeSO}_{4}\left(\mathrm{pH} \mathrm{7.4)}\right.$ at $37^{\circ} \mathrm{C}$ for 6 to $8 \mathrm{~h}$ with dialysate changes every $2 \mathrm{~h}$ (17). Oxidation was stopped by the addition of
EDTA (100 $\mu \mathrm{M}$, final concentration), the LDL was sterilized by passage through a $0.22-\mu \mathrm{m}$ filter, and total cholesterol and TBA reactivity were determined. Phospholipase $\mathrm{A}_{2}\left(\mathrm{PLA}_{2}\right)$-modified lipoprotein was prepared by incubating LDL ( 2 to $3 \mathrm{mg}$ cholesterol) with bee venom $\mathrm{PLA}_{2}$ attached to agarose beads (Sigma Chemical Co.) in 100 $\mathrm{mM}$ Hepes ( $\mathrm{pH} 8.5$ ) containing $3 \mathrm{mM} \mathrm{CaCl}_{2}$ and $25 \mu \mathrm{M}$ butylated hydroxytoluene in a shaking water bath at $37^{\circ} \mathrm{C}(36)$. In experiments where maximal formation of lysoPC was the desired end-point, LDL was incubated with $5 \mathrm{U}$ of immobilized $\mathrm{PLA}_{2}$ in a total volume of $1 \mathrm{ml}$ for $1 \mathrm{~h}$. In experiments in which a range of lysoPC amounts was required, $0.25 \mathrm{U}$ of enzyme was added in a total volume of $1.7 \mathrm{ml}$ for times ranging from 10 to 240 min (this amount of enzyme and time intervals were shown in preliminary studies to generate a broad and consistent range of LDL lysoPC levels under these conditions). The enzyme was removed by centrifugation and the LDL was concentrated to its original volume using a Centricon-10 (Amicon, Danvers, MA), and sterilized by filtration.

Extraction and fractionation of lipids from oxidized $L D L$. Lipids were extracted from oxidized LDL using chloroform:methanol (37), dried under $\mathrm{N}_{2}$, redissolved in the same solvent, and stored at $-80^{\circ} \mathrm{C}$. The final concentration of the solvent in the culture medium was $<0.5 \%$ by volume in all the experiments where lipid was delivered in solvent. Lipid-free controls containing this amount of solvent were tested and did not alter EC migration. Extracted lipids were dried under $\mathrm{N}_{2}$, dissolved (at $4 \mathrm{mg} / \mathrm{ml}$ ) in $n$-hexane:2-propanol:water (39:52:9, $\mathrm{vol} / \mathrm{vol})$, and fractionated $(1 \mathrm{ml} / \mathrm{min})$ on a silica HPLC column (Lichrosorb SI-60 $10 \mu \mathrm{m} ; 250 \times 4.6 \mathrm{~mm}$; Alltech Associates Inc., Deerfield, IL) using the same solvent under isocratic conditions (38). Eluted lipids were detected by absorbance at $206 \mathrm{~nm}$. Lysophospholipids containing saturated fatty acids have negligible absorbance at this wavelength, and were detected by an alternate method: the HPLC fractions were subjected to silica gel thin layer chromatography using chloroform:acetone:methanol:acetic acid:water (60:80:20: 20:10, vol/vol) and visualized with Coomassie brilliant blue (39).

Measurement of endothelial cell motility. EC migration was measured by the "razor wound" method essentially as described $(14,17)$. Confluent EC in 12-well tissue culture clusters were made quiescent by replacing the medium with serum-free Dulbecco's modified Eagles medium (Sigma Chemical Co.) containing $1 \mathrm{mg} / \mathrm{ml}$ gelatin for at least $24 \mathrm{~h}$ before use. The cultures were wounded with a razor and medium containing gelatin $(1 \mathrm{mg} / \mathrm{ml})$ plus test lipids were added in a total volume of $0.5 \mathrm{ml}$. Wound-induced migration was terminated after $22-24 \mathrm{~h}$ by fixing and staining with modified Wright-Giemsa stain (Sigma Chemical Co.). A 256-gray level, $640 \times 480$ pixel image of the cultures was captured by a digital charged couple device camera mounted on a phase-contrast microscope. Image analysis was done using the "Image" software package provided by Dr. Wayne Rasband, National Institutes of Health. In each well, two random fields, each consisting of $1500 \mu \mathrm{m}$ length of wound, were chosen and the number of cells that crossed the origin line was determined. The data from duplicate wells were expressed as the mean and standard error of the number of migrating cells per $3000 \mu \mathrm{m}$ of wound length. All experiments were done two or more times, and representative results are shown.

\section{Results}

Lysophosphatidylcholine inhibits endothelial cell migration. Our previous work indicated that metal ion-oxidized LDL blocks EC movement, and that about half of this activity was recovered in a solvent extract (17). We tested the antimigratory activity of lysoPC, a major component of oxidized LDL with several known cellular activities. Micrographs of EC taken after a 22-h migration period clearly showed that 1-palmitoyl lysoPC (but not PC) inhibited wound-stimulated EC movement in a concentration-dependent manner and without causing apparent morphological injury (Fig. 1). Quantitation 


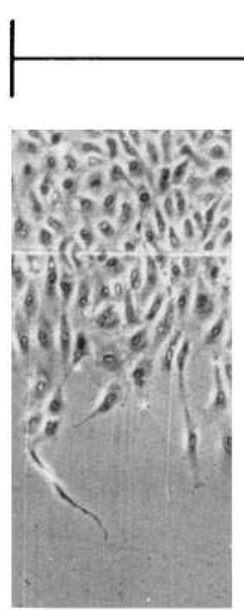

0

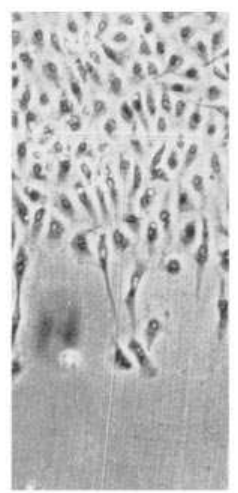

4

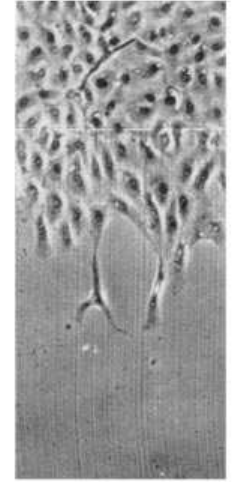

8
LysoPC

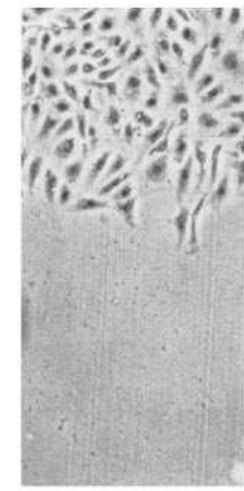

12

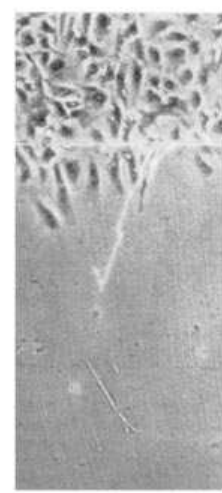

16

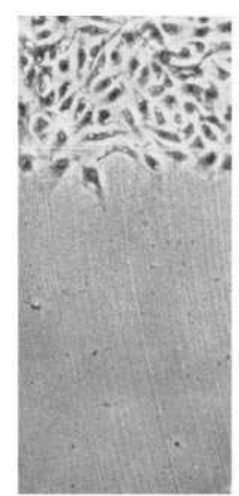

20

\section{Concentration of Phospholipid $(\mu \mathrm{M})$}

Figure 1. Inhibition of EC migration by lysoPC. Confluent, quiescent cultures of bovine aortic EC were incubated with 1-palmitoyl lysoPC (dissolved in medium) and egg PC (sonicated liposomes) at indicated concentrations. Migration was terminated after $22 \mathrm{~h}$ by addition of modified Wright-Giemsa stain, and micrographs taken by phase contrast microscopy.
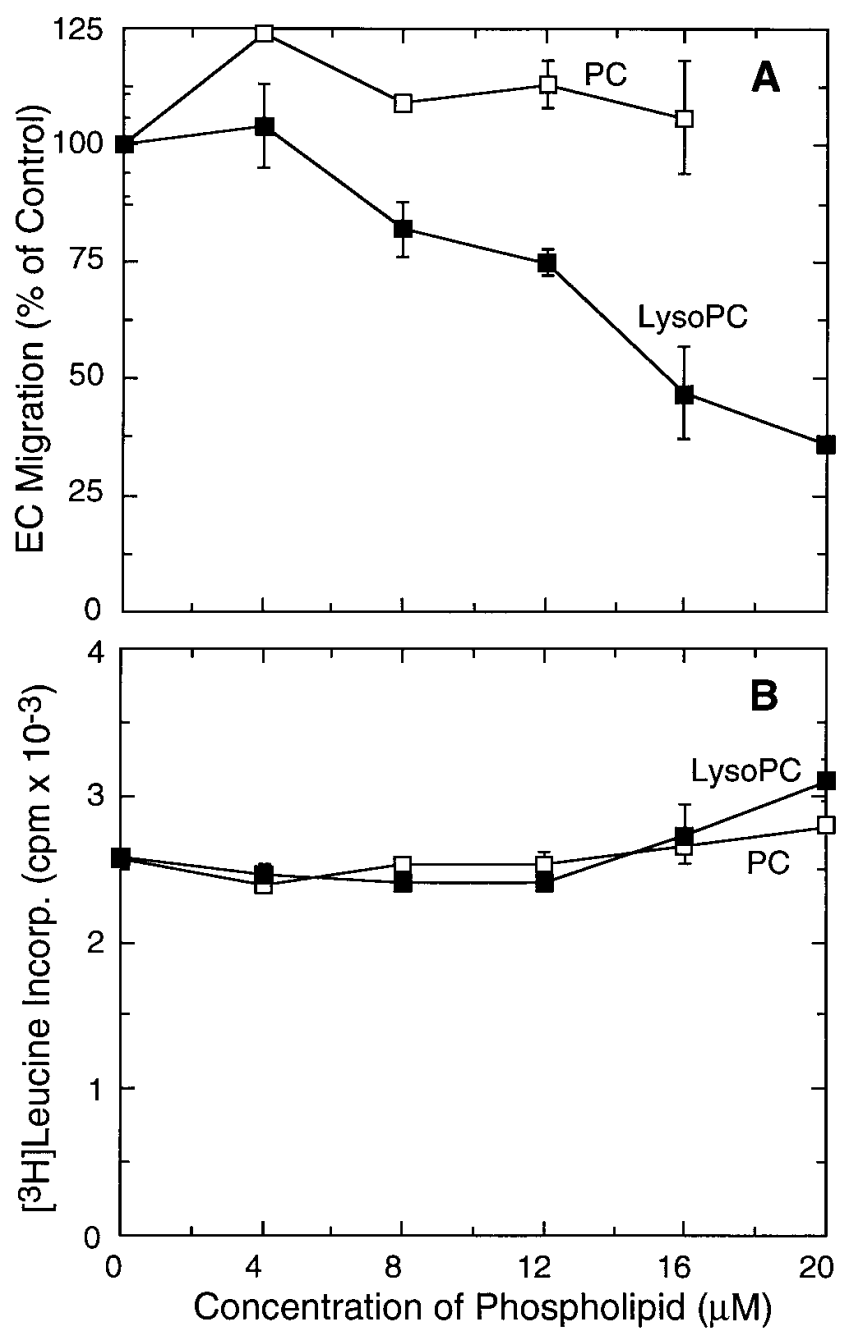

Figure 2. Quantitation of antimigratory activity of lysoPC and its effect on EC viability. EC were incubated with egg PC ( $\square$ ) and 1-palmitoyl lysoPC ( $\mathbf{\square})$ for $22 \mathrm{~h}$ as described in Fig. 1. (A) The number of mi- of this result as the number of migrating EC gave a half-maximal inhibitory concentration at $12-15 \mu \mathrm{M}$, and near maximal inhibition at $20 \mu \mathrm{M}$, concentrations below the critical micellar concentration of 40-50 $\mu \mathrm{M}$ (40) (Fig. 2 A). Similar results were obtained in multiple isolates of bovine aortic EC. The absence of a cytotoxic effect of lysoPC was shown by determination of cellular protein synthesis by incorporation of $\left[{ }^{3} \mathrm{H}\right]$ leucine into cellular protein (Fig. 2 B). The lack of toxicity of lysoPC was verified by the absence of trypan blue-stained cells (not shown) and by continuous time-lapse videomicrography during which death and subsequent detachment of cells was not seen. The inhibitory activity of 1-palmitoyl lysoPC was completely reversed within $24 \mathrm{~h}$ after removal of the lipid, further verifying that the inhibition of migration was not due to cell injury or death (Fig. 3). To show that lysoPC specifically inhibited migration rather than proliferation the effect of hydroxyurea, an inhibitor of EC proliferation was used. At $1 \mathrm{mM}$ hydroxyurea, a concentration that completely blocks proliferation of bovine aortic EC (14), lysoPC (20 $\mu \mathrm{M})$ inhibited cell migration by $69 \%$ (mean of three experiments), showing that lysoPC specifically inhibits EC movement.

The influence of lysophospholipid structure on antimigratory activity. The specific structural attributes, i.e., 1-position fatty acid chain length and degree of saturation in addition to head group, that influence lysolipid antimigratory activity were investigated. Examining a series of lysoPC's containing saturated fatty acids, 1-stearoyl (C18:0) lysoPC was seen to have approximately the same potent antimigratory activity as 1-palmitoyl (C16:0) lysoPC (Fig. 4 A). In contrast, lysoPC's containing shorter saturated fatty acids (C10:0, C12:0, and

grating cells in duplicate wells (mean \pm standard error) was quantitated by computer-assisted particle analysis. Cell migration in control wells was $344 \pm 15$ cells per $3000 \mu \mathrm{m}$ of wound. (B) In parallel duplicate wells, $\left[{ }^{3} \mathrm{H}\right]$ leucine $(1 \mu \mathrm{Ci} / \mathrm{ml})$ was added during the final $2 \mathrm{~h}$ of the incubation, and cellular protein synthesis measured as trichloroacetic acid-precipitable protein. 


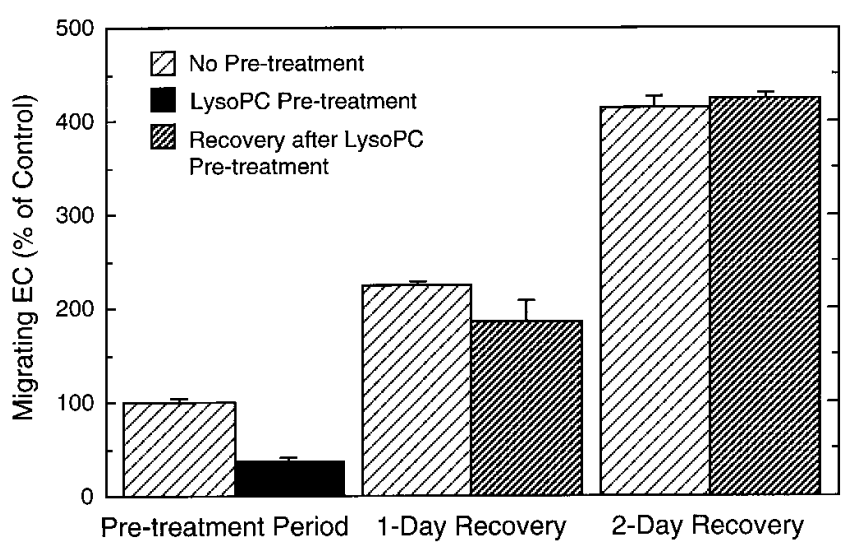

Figure 3. Reversibility of antimigratory activity of lysoPC. Confluent EC cultures were incubated for 1-day in the presence or absence of 1-palmitoyl lysoPC $(18 \mu \mathrm{M})$, the medium replaced with lysoPC-free medium, and cell migration allowed to continue for two additional days. After each day duplicate EC cultures were fixed and stained, and cell migration was determined. The number of migrating cells in cultures of untreated EC (light hatched bars), EC treated with lysoPC (solid bar), and EC treated with lysoPC followed by lysoPC-free medium (heavy hatched bars) are shown. Cell migration in control wells was $408 \pm 18$ cells.

C14:0) were almost completely without effect. The degree of saturation of the fatty acid was also a critical determinant of activity. LysoPC containing 1-position saturated fatty acids (C16:0 and C18:0) were slightly more active than lysoPC with a monounsaturated fatty acid (C18:1), and lysoPC containing polyunsaturated fatty acids (C18:2 and C18:3) were completely ineffective (Fig. $4 \mathrm{~B}$ ). The antimigratory activity of the satu- rated, long chain fatty acids was not due to contaminating free fatty acids or intracellular release of fatty acids (by cellular lysophospholipase activity) since free fatty acids at the same concentration were not antimigratory (not shown).

To investigate the role of lysolipid head group, the antimigratory activity of a series of 1-palmitoyl lysophospholipids was examined. LysoPI was found to be slightly more potent than lysoPC, and lysoPG and lysoPS had about the same activity as lysoPC (Fig. 4 C). LysoPE and 1-palmitoyl monoglyceride were ineffective while lysoPA was unique among all lysolipids tested in that it stimulated EC movement by up to $50 \%$.

Antimigratory activity of phospholipase $A_{2}$-modified LDL. The cellular delivery of purified lysophospholipids as emulsions is almost certainly not equivalent (in terms of amounts delivered, utilization of cellular receptors, intracellular localization and metabolism, etc.) to the delivery of the same lipids within intact lipoproteins. We therefore investigated the antimigratory activity of lysoPC generated within LDL itself by treatment of the particle with $\mathrm{PLA}_{2}$. Native LDL was treated with $5 \mathrm{U}$ of immobilized bee venom $\mathrm{PLA}_{2}$ as described in Methods. Lipoprotein PC was converted almost completely to lysoPC as determined by Coomassie brilliant blue staining of lipid extracts after thin layer chromatography; the final amount of lysoPC was $335 \mu \mathrm{g} / \mathrm{mg}$ LDL cholesterol. PLA -modified $_{2}$ LDL inhibited EC migration in a dose-dependent manner with a potency and maximal activity comparable to LDL oxidized by incubation with $5 \mu \mathrm{M} \mathrm{FeSO}_{4}$ (and containing $239 \mu \mathrm{g}$ lysoPC/mg LDL cholesterol) (Fig. 5). Since Fe-oxidized LDL has about the same antimigratory activity as $\mathrm{PLA}_{2}$-treated LDL, while containing less lysoPC, it is possible that the former may contain additional non-lysoPC-like inhibitors (see below). Untreated LDL and LDL mock-treated with phospholipase-free agarose beads did not inhibit EC movement.

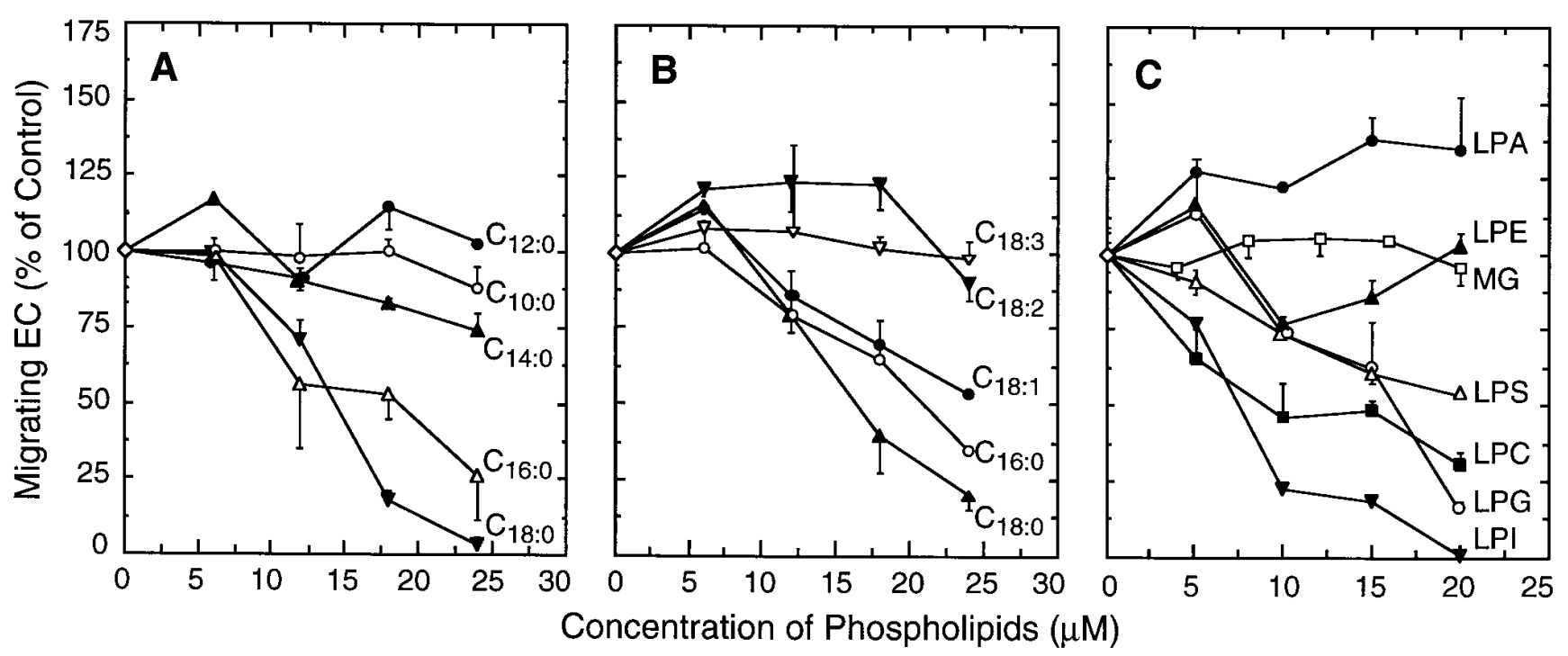

Figure 4. Effect of lysolipid structure on antimigratory activity. Bovine aortic EC cultures were wounded and incubated for $22 \mathrm{~h}$ with lysolipids at the concentrations shown; control wells not containing lysolipids are indicated $(\diamond)$. $(A)$ Wounded EC were treated with a homologous series of lysoPC's containing 1-position saturated fatty acids: 1-decanoyl lysoPC $\left(\mathrm{C}_{10: 0}, \bigcirc\right)$; 1-lauroyl lysoPC $\left(\mathrm{C}_{12: 0}, \bullet\right)$; 1-myristoyl lysoPC $\left(\mathrm{C}_{14: 0}, \mathbf{\Delta}\right)$; 1-palmitoyl lysoPC $\left(\mathrm{C}_{16: 0}, \triangle\right)$; and 1-stearoyl lysoPC $\left(\mathrm{C}_{18: 0}, \boldsymbol{\nabla}\right)$. (B) Wounded EC were treated with lysoPC's containing 1-position fatty acids of varying degrees of unsaturation: 1-palmitoyl lysoPC $\left(\mathrm{C}_{16: 0}, \bigcirc\right)$; 1-stearoyl lysoPC $\left(\mathrm{C}_{18: 0}, \mathbf{\Delta}\right)$; 1-oleoyl lysoPC $\left(\mathrm{C}_{18: 1}, \boldsymbol{\bullet}\right)$; 1-linoleoyl lysoPC $\left(\mathrm{C}_{18: 2}\right.$, $\nabla)$; and 1-linolenoyl lysoPC $\left(\mathrm{C}_{18: 3}, \nabla\right)$. (C) Wounded $\mathrm{EC}$ were treated with 1-palmitoyl lysolipids containing various head groups: lysoPI (LPI,

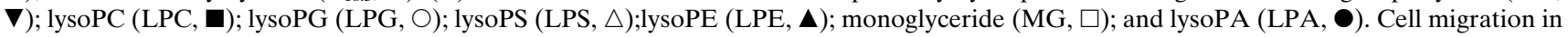
control wells was $508 \pm 15,450 \pm 21$, and $271 \pm 12$ cells in $A, B$, and $C$, respectively. 


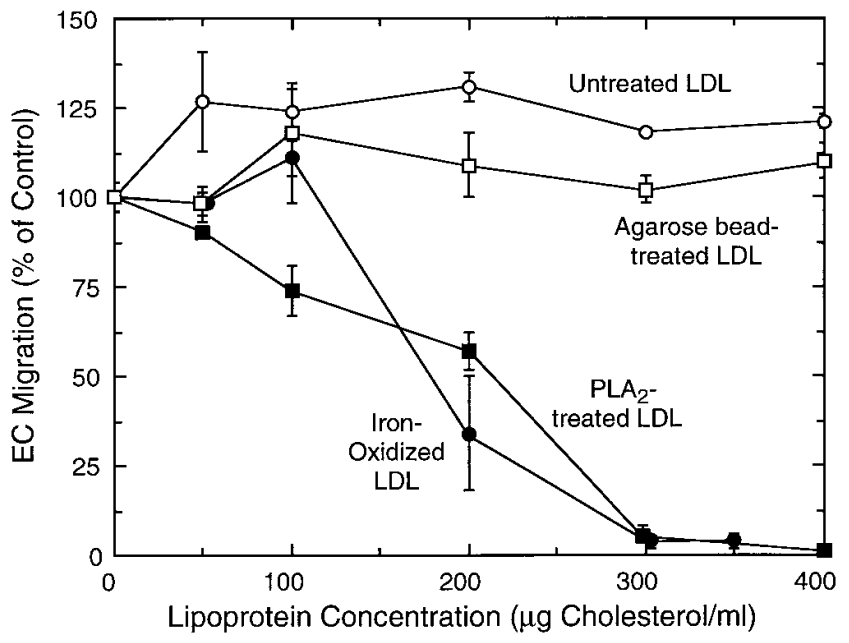

Figure 5. Antimigratory activity of phospholipase $\mathrm{A}_{2}$-treated LDL. Native LDL ( $2.5 \mathrm{mg}$ cholesterol) was modified by incubation with bee venom $\mathrm{PLA}_{2}(5 \mathrm{U})$ immobilized on agarose beads for $1 \mathrm{~h}$ at $37^{\circ} \mathrm{C}$ as described in Methods. As a control, LDL was similarly treated with phospholipase-free agarose beads. LDL was oxidized by incubation with $5 \mu \mathrm{M} \mathrm{FeSO}_{4}$ for $6 \mathrm{~h}$ at $37^{\circ} \mathrm{C}$ (final TBA reactivity was approximately $8 \mathrm{nmol} \mathrm{MDA} / \mathrm{mg}$ cholesterol). Untreated LDL (O), agarose bead-treated LDL ( $\square), \mathrm{PLA}_{2}$-modified $\operatorname{LDL}(\mathbf{\square})$, and Feoxidized LDL $(\bullet)$ were incubated with wounded EC cultures for $22 \mathrm{~h}$ and EC migration determined. Cell migration in control wells was $224 \pm 2$ cells.

To more directly evaluate the role of lysoPC in the antimigratory activity of $\mathrm{PLA}_{2}$-treated lipoprotein, LDL was variably modified by incubation with $\mathrm{PLA}_{2}$ for several times up to $4 \mathrm{~h}$, and the activity was quantitatively compared to lysoPC content. As expected, the appearance of lysoPC correlated with the disappearance of PC (Fig. 6). Furthermore, the antimigratory activity directly correlated with lysoPC content providing further evidence that lysoPC within LDL was an effective inhibitor.

Antimigratory activity of lysoPC fraction of iron-oxidized $L D L$. To investigate the potential role of lysoPC in the antimigratory activity of oxLDL, the amount of lysoPC in Fe-oxidized LDL was quantitated by thin layer chromatography of solvent extracts and densitometry of the lysoPC fractions after staining with Coomassie Brilliant Blue (and comparison to authentic standards). Oxidized LDL contained 200-250 $\mu \mathrm{g}$ of lysoPC per $\mathrm{mg}$ of LDL cholesterol, an amount within the range reported by others $(37,41)$. LysoPC was absent or present in only trace amounts in native LDL. In our previous work the maximal antimigratory activity of oxidized LDL was observed at about $400 \mu \mathrm{g}$ cholesterol/ml (17), a lipoprotein concentration equivalent to $160-200 \mu \mathrm{M}$ of lysoPC. Thus the amount of lysoPC present in Fe-oxidized LDL is more than sufficient to account for the antimigratory activity of the parent lipoprotein.

The antimigratory activity of the lipid component of Feoxidized LDL, was tested after extraction with chloroform: methanol (37) and sonication in medium. The solvent-extracted fraction contained substantial antimigratory activity, but it was significantly less than the intact particle (Fig. $7 \mathrm{C}$ ). This was consistent with our previous report that an acetone extract of Fe-oxidized LDL contained about half of the activity of the unextracted lipoprotein (17). Incomplete recovery in solvent

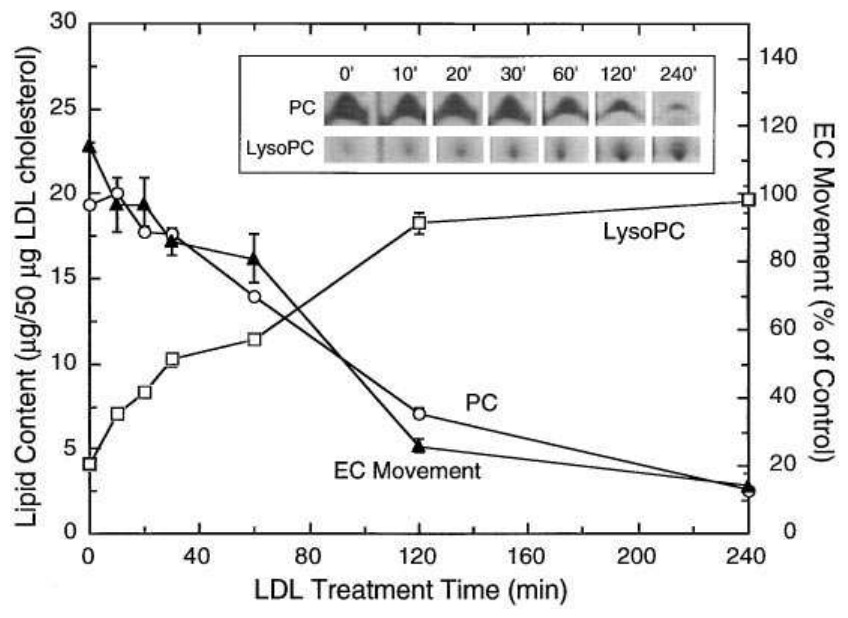

Figure 6. Correlation of lysoPC and antimigratory activity in phospholipase $\mathrm{A}_{2}$-treated LDL. Native LDL (3 mg cholesterol) was modified with bee venom $\operatorname{PLA}_{2}(0.25 \mathrm{U})$ immobilized on agarose beads for up to $4 \mathrm{~h}$. The lipids from LDL containing $50 \mu \mathrm{g}$ cholesterol were extracted with chloroform:methanol, subjected to silica gel thin layer chromatography, and visualized with Coomassie brilliant blue (inset). The amounts of lysoPC and PC were quantitated by densitometry and comparison to authentic phospholipid standards. Wounded EC cultures were incubated with PLA 2 -modified LDL (300 $\mu \mathrm{g}$ cholesterol $/ \mathrm{ml}$ ) for $22 \mathrm{~h}$ and EC migration ( $\mathbf{\Lambda})$ compared with the amount of lysoPC $(\square)$ and PC $(\bigcirc)$ in the modified lipoprotein. Cell migration in control wells was $226 \pm 9$ cells.

extracts was shown previously for several distinct cellular activities of oxidized LDL including inhibition of growth factor and cytokine expression by several cell types (42-44); the incomplete recovery could be due to the incomplete extraction of the lipid inhibitor(s), the presence of non-extractable inhibitors, or the lower activity of the inhibitor lipid when delivered to cells in emulsified extracts rather than in an intact lipoprotein. The solvent extract of oxidized LDL was fractionated by normal phase (silica) HPLC (Fig. 7, $A$ and $B$ ). The absorbance profile at $206 \mathrm{~nm}$ indicates that a substantial amount of the PC in LDL is modified during the oxidation procedure. An absorbance peak corresponding to the appearance of lysoPC was not present since lysoPC's containing 1-position saturated and monounsaturated fatty acids have negligible absorbance at this wavelength (data not shown). To identify lysoPC and other lipid components, 10-min fractions were taken, dried under $\mathrm{N}_{2}$, subjected to silica gel thin layer chromatography, and visualized with Coomassie brilliant blue. Only fractions V and VI (corresponding to elution times between 40 and $60 \mathrm{~min}$ ) contained lipids that comigrated with authentic lysoPC as shown by HPLC chromatography of lysoPC standards, (not shown), and by comparison of the fractions to 1-palmitoyl lysoPC thin layer chromatography followed by Coomassie brilliant blue staining (Fig. $7 \mathrm{~B}$, inset). The corresponding fractions in LDL did not contain detectable amounts of lysoPC (Fig. $7 \mathrm{~A}$, inset).

The antimigratory activity of the HPLC fractions of LDL and oxidized LDL was tested. HPLC fractions were dried under $\mathrm{N}_{2}$, emulsified in medium using a bath sonicator, and added to wounded EC cultures (Fig. 7 C). The fractions of oxidized LDL containing lysoPC (fractions V and VI) are potent inhibitors of EC movement with an activity essentially the same as an equivalent amount of intact oxidized LDL, and surpassing the activity of the whole extract. In a positive control 

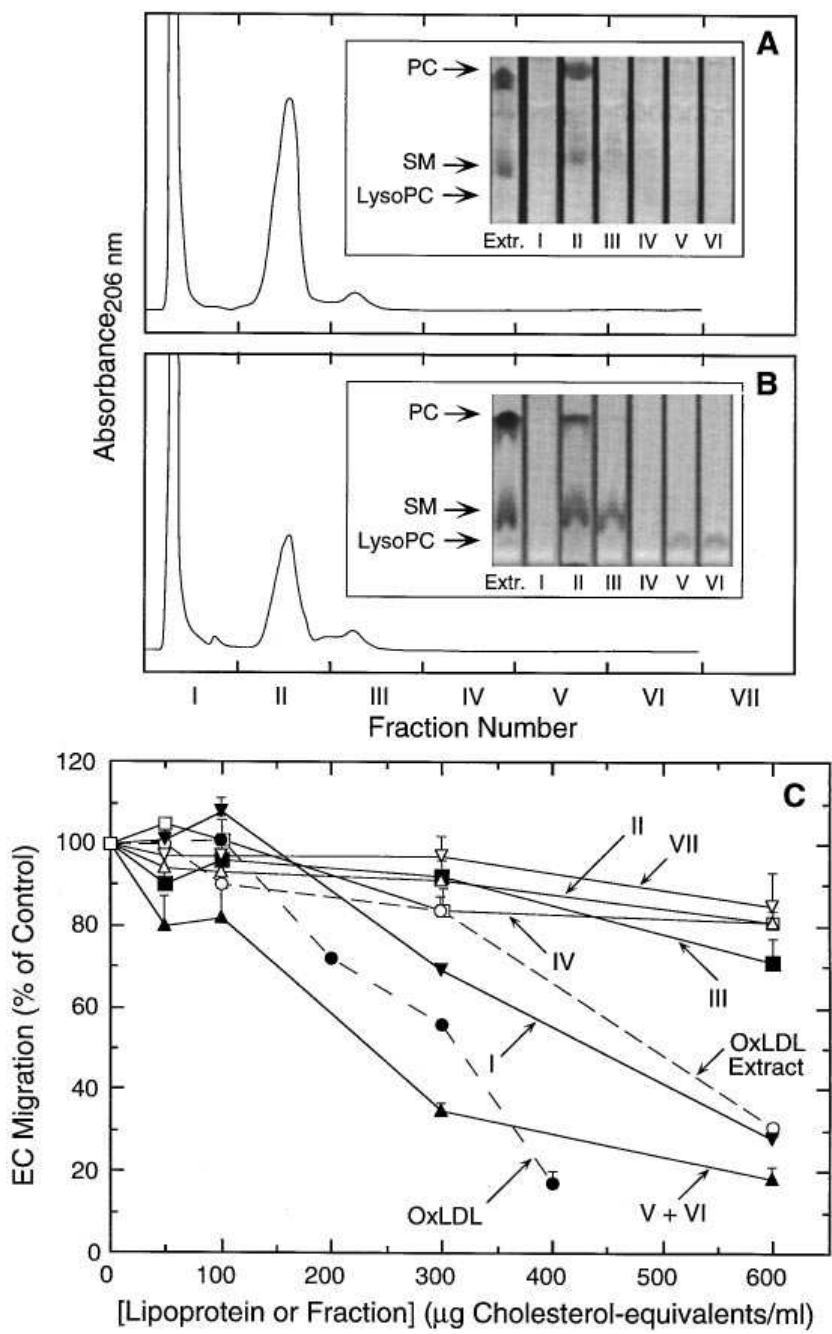

Figure 7. Identification of antimigratory lipid fractions by normal phase HPLC. Lipids were extracted from native and Fe-oxidized LDL described in Methods and subjected to normal phase HPLC on a silica column. Fractions were collected at $10 \mathrm{~min}$ intervals, dried under $\mathrm{N}_{2}$, chromatographed on silica gel thin layer chromatography plates (next to PC, lysoPC and sphingomyelin [SM] standards), and visualized with Coomassie brilliant stain. (A) Absorbance $(206 \mathrm{~nm})$ trace from an extract of native LDL (600 $\mu \mathrm{g}$ of cholesterol). Inset, thin layer chromatogram of native LDL extract (Extr.) and fractions I through VI. $(B)$ Absorbance trace from an extract of Fe-oxidized LDL (600 $\mu \mathrm{g}$ cholesterol). Inset, thin layer chromatogram of oxidized LDL extract and fractions I through VI. (C) HPLC fractions from extract of Fe-oxidized LDL were dried under $\mathrm{N}_{2}$ and emulsified by sonication in medium. Confluent EC cultures were wounded and incubated with oxidized LDL (OxLDL, $\bullet)$, the unfractionated solvent extract (O), and HPLC fractions I ( $\boldsymbol{\nabla})$, II $(\triangle)$, III $(\boldsymbol{\square})$, IV ( $\square)$, V + VI $(\boldsymbol{\Delta})$, and VII $(\nabla)$ for $22 \mathrm{~h}$, and cell migration was determined. Cell migration in control wells was $535 \pm 10$ cells.

experiment, $\mathrm{PLA}_{2}$-treated LDL was extracted and fractionated by HPLC by the same methods; both lysoPC (by thin layer chromatography and Coomassie blue staining) and antimigratory activity appeared in fractions V and VI only, thus confirming the utility of the method and the activity of lysoPC. Fractions V and VI from native LDL did not have antimigratory activity (not shown). A significant amount of the antimigratory activity in oxidized LDL lipids was consistently seen in fraction I. This lipid fraction is likely to contain cytotoxic sterol derivatives including $7 \beta$-hydroperoxycholestenol, the major cytotoxin in oxidized LDL (30). This fraction was not toxic (by morphological criteria) in our experiments, possibly due to the fact that the cytotoxic lipids studied to date are most effective on proliferating cells, and the unstimulated, confluent cells in serum-free medium used in our experiments should be less susceptible to their action. 7 $\beta$-Hydroperoxycholestenol and its $7 \alpha$ - stereoisomer were found to completely lack antimigratory activity (at concentrations equivalent to those present in oxidized LDL), and the identity of the specific antimigratory lipid(s) in fraction I is yet to be identified. The oxidized LDL fractions containing PC and sphingomyelin (fractions II and III) and two fractions not containing Coomassie-detectable lipids were almost completely inactive (as was a solvent control). In summary, these results show that a majority of the antimigratory activity of oxidized LDL is either lysoPC or a structurally related, co-migrating lipid.

\section{Discussion}

During metal ion-mediated LDL oxidation, lysoPC is thought to be formed from PC by an endogenous phospholipase $\mathrm{A}_{2}$ activity specific for phospholipids containing oxidatively-modified chains in the 2-position (45). Our results indicate that lysoPC is the lipid primarily responsible for oxidized LDL-mediated inhibition of $\mathrm{EC}$ movement. This conclusion is supported by the antimigratory activity of lysoPC added either as vesicles of purified lipid or within $\mathrm{PLA}_{2}$-modified LDL. Furthermore, Fe-oxidized LDL contains amounts of lysoPC consistent with antimigratory activity, and the majority of the activity of a solvent extract of oxidized LDL is found in an HPLC fraction that co-migrates with authentic lysoPC. Our finding that lysoPC has a predominant role in the function of oxidized LDL is consistent with observations of several laboratories investigating multiple vascular processes. LysoPC is a chemoattractant for monocytes (46) and induces leukocyte adhesion molecules (40); lysoPC suppresses endothelin-1 secretion by EC (37) but impairs endothelium-dependent relaxation of vessel strips (47); lysoPC induces transcription of platelet-derived growth factor A- and B-chains and heparin-binding epidermal growth factor-like protein (48) and enhances smooth muscle cell (49) and macrophage proliferation (50). In several of these studies lysoPC was shown to be primarily or entirely responsible for the cellular activity of oxidized LDL $(37,40,46,47)$.

Investigation of the antimigratory activity of various lysophospholipids shows that specific structural features profoundly influence activity. The length of the fatty acid is an important regulatory factor since lysoPC containing sn-1position saturated fatty acid chains containing 16 or 18 carbons are highly inhibitory, while those containing 10,12 , or 14 carbons are ineffective. The relative activities are similar to those reported for chemoattraction of lymphoblastic (51) and monocytic (46) cells by lysoPC. Similarly, lysoPC containing long-chain saturated fatty acids preferentially stimulate $\left(\mathrm{Ca}^{2+}\right.$, $\mathrm{Mg}^{2+}$ )-ATPase activity of human erythrocyte membranes (52). Our results also indicate that lysoPC's containing saturated and monounsaturated fatty acids have much more antimigratory activity than those containing polyunsaturated fatty acids, a finding not seen in all cell processes influenced by lysolipids (52). 
Our results also show that lysolipid head group is a determinant of antimigratory activity. Specifically, the activity of lysoPI is much higher than lysoPC, lysoPG, and lysoPS, which in turn are higher than lysoPE and monoacylglycerol. This order of activity is consistent with that seen for chemoattraction of human monocytes (46). It is noteworthy that the same lipids that stimulate movement of monocytes, T-lymphocytes, and smooth muscle cells (23), inhibit movement of EC. In contrast, Kume et al. (40) have shown that the induction of EC adhesion molecules by lysoPE (and lysoPC) is significantly higher than by lysoPI (and lysoPS). Taken together these results again suggest that there are differential biological activities of specific lysophospholipids. The results of Kume et al. (40) are consistent with a charge-dependence of lysolipid activity (phospholipids without net charge having more activity than negative phospholipids), but the same conclusion cannot be made for antimigratory activity. In contrast, our data are consistent with a mechanism in which head group molecular size is the primary determinant of activity since the size of the head group of the most inhibitory lipid, lysoPI $(257 \mathrm{D})$, is greater than that for the moderately inhibitory lysoPC (182 D), lysoPG (170 D), and lysoPS $(183 \mathrm{D})$, which are greater than that for the inactive lysolipids, lysoPE (140 D) and monoacylglycerol (no head group). According to one possible mechanism, a large head group spatially constrains lysolipid packing and adversely affects membrane function. Similar results showing a correlation between lysophospholipid head group size (and not charge) on ion channel function have been previously reported (53). In contrast to all other lysolipids tested, which either inhibit or do not affect EC movement, lysoPA is unique since it enhances cell movement in our assay system. This finding differs somewhat from the report of Jalink et al. (54) in which lysoPA is shown to be a chemoattractant for Dictyostelium discoideum (when applied as a gradient), but unlike our observations with $\mathrm{EC}$, it lacks chemokinetic activity in that system.

The mechanism(s) by which lysoPC inhibits EC motility is not known. Although lysoPC at concentrations above its critical micellar concentration is cytotoxic (55), in the observed suppression of EC migration is not due to lethal cell injury since: (a) inhibition is observed at lysolipid concentrations below the critical micellar concentration; $(b)$ cellular protein synthesis is unaffected; and (c) the inhibition is completely reversed by removal of the lipid. LysoPC may exert antimigratory activity by multiple mechanisms including interference with: growth factor receptor activity (lysoPC blocks the promigratory activity of basic fibroblast growth factor, not shown), intracellular signaling pathways, assembly of cytoskeletal structures involved in cell motility, and activity of proteases that degrade extracellular matrix molecules and are required for cell movement. Recent reports indicate that lysoPC-mediated EC dysfunction $(56,57)$ involves the activation of protein kinase $\mathrm{C}$. In other studies lysoPC and pertussis toxin have been shown to inhibit vessel relaxation by similar mechanisms, suggesting that lysoPC may interfere with a G-protein-mediated signaling pathway (58). This mechanism is consistent with our previous report that basic fibroblast growth factor-stimulated movement of EC requires the activity of a pertussis toxin-sensitive G-protein (59).

The physiological role for the antimigratory activity of lysolipids has not been examined. However, the presence of lysoPC in physiological compartments exposed to vascular endothelium in vivo has been demonstrated, e.g., lysoPC is a nor- mal constituent of plasma (60) and hyperlipemic LDL contains elevated amounts of lysoPC (61). Furthermore, the amount of lysoPC in atherosclerotic lesions in humans and experimental animals is higher than in uninvolved tissues (61-63). Oxidatively-modified LDL is a likely source of sub-endothelial lysoPC since lesions contain lipoproteins resembling oxidized LDL (19). The finding that lysoPC's with long-chain, saturated fatty acids are antimigratory is consistent with the inhibitory activity of lysolipids in oxidized LDL since the $s n-1$ position of PC in human LDL is enriched with palmitic ( $~ 60 \%$ of total) and stearic $(\sim 25 \%$ of total) acids $(64,65)$. Similarly, the lysolipid component of LDL and its oxidatively-modified counterpart is primarily the highly active lysoPC with very low amounts of the less active lysoPS and lysoPE (46).

The presence of lysoPC in the subendothelial space invites speculation that its antimigratory activity may have a role in the limited re-endothelialization observed in injured blood vessels and in arterial grafts in vivo (66). We propose that the following events occur. After vessel injury, the tissue below the regenerating endothelium preferentially accumulates lipoproteins $(67,68)$ which become oxidatively modified by cell-mediated processes $(69,70)$ and trapped by their high affinity for collagen and other matrix molecules (71). This process leads to an increase in the local concentration of lysoPC which may subsequently limit the regeneration of endothelium. Further studies are necessary to understand the molecular mechanisms by which lysoPC inhibits EC migration and define its role in the $\mathrm{EC}$ regeneration process.

\section{Acknowledgments}

We thank Alan Prok and Christine McKenzie for excellent technical assistance and James Lang for photography. Guy Chisolm is acknowledged for helpful discussions and for $7 \alpha$ - and $7 \beta$-hydroperoxycholestenol.

This work was supported by National Institutes of Health grants HL-41178, HL-52692, and HL54519, by an unrestricted grant for cardiovascular research from Berlex Biosciences, and by a Fellowship (to G. Murugesan) from the American Heart Association, Northeast Ohio Affiliate.

\section{References}

1. Schwartz, S.M., C.M. Gajdusek, and S.C. Selden. 1981. Vascular wall growth control: the role of the endothelium. Arteriosclerosis. 1:107-126.

2. Coomber, B.L., and A.I. Gotlieb. 1990. In vitro endothelial wound repair. Interaction of cell migration and proliferation. Arteriosclerosis. 10:215-222.

3. Ausprunk, D.H., and J. Folkman. 1977. Migration and proliferation of endothelial cells in preformed and newly formed blood vessels during tumor angiogenesis. Microvasc. Res. 14:53-65.

4. Zetter, B.R. 1980. Migration of capillary endothelial cells is stimulated by tumour-derived factors. Nature (Lond.). 285:41-43

5. Montesano, R., and L. Orci. 1987. Phorbol esters induce angiogenesis in vitro from large-vessel endothelial cells. J. Cell. Physiol. 130:284-291.

6. Connolly, D.T., D.M. Heuvelman, R. Nelson, J.V. Olander, B.L. Eppley, J.J. Delfino, N.R. Siegel, R.M. Leimgruber, and J. Feder. 1989. Tumor vascular permeability factor stimulates endothelial cell growth and angiogenesis. J. Clin. Invest. 84:1470-1478.

7. Rosen, E.M., W. Carley, and I.D. Goldberg. 1990. Scatter factor regulates vascular endothelial cell motility. Cancer Invest. 8:647-650.

8. Koch, A.E., P.J. Polverini, S.L. Kunkel, L.A. Harlow, L.A. DiPietro, V.M. Elner, S.G. Elner, and R.M. Strieter. 1992. Interleukin-8 as a macrophage-derived mediator of angiogenesis. Science (Wash. DC). 258:1798-1801.

9. Bjornsson, T.D., M. Dryjski, J. Tluczek, R. Mennie, J. Ronan, T.N. Mellin, and K.A. Thomas. 1991. Acidic fibroblast growth factor promotes vascular repair. Proc. Natl. Acad. Sci. USA. 88:8651-8655.

10. Lindner, V., R.A. Majack, and M.A. Reidy. 1990. Basic fibroblast growth factor stimulates endothelial regrowth and proliferation in denuded arteries. J. Clin. Invest. 85:2004-2008. 
11. Keegan, A., C. Hill, S. Kumar, P. Phillips, A. Schor, and J. Weiss. 1982. Purified tumour angiogenesis factor enhances proliferation of capillary, but not aortic, endothelial cells in vitro. J. Cell Sci. 55:261-276.

12. Gotlieb, A.I., W. Spector, K. Desai, and G. Steiner. 1983. The effect of low density lipoprotein on in vitro porcine endothelial cell reendothelialization. Artery. 12:132-144.

13. Tauber, J.P., D. Goldminz, and D. Gospodarowicz. 1981. Up-regulation in vascular endothelial cells of binding sites of high density lipoprotein induced by 25-hydroxycholesterol. Eur. J. Biochem. 119:327-339.

14. Murugesan, G., G. Sa, and P.L. Fox. 1994. High-density lipoprotein stimulates endothelial cell movement by a mechanism distinct from basic fibroblast growth factor. Circ. Res. 74:1149-1156.

15. Madri, J.A., M.A. Reidy, O. Kocher, and L. Bell. 1989. Endothelial cell behavior after denudation injury is modulated by transforming growth factor$\beta 1$ and fibronectin. Lab. Invest. 60:755-764.

16. Pepper, M.S., N. Ferrara, L. Orci, and R. Montesano. 1995. Leukemia inhibitory factor (LIF) inhibits angiogenesis in vitro. J. Cell Sci. 108:73-83.

17. Murugesan, G., G.M. Chisolm, and P.L. Fox. 1993. Oxidized low density lipoprotein inhibits the migration of aortic endothelial cells in vitro. J. Cell Biol. 120:1011-1019.

18. Boissonneault, G.A., Y. Wang, and B.H. Chung. 1995. Oxidized lowdensity lipoproteins delay endothelial wound healing: Lack of effect of vitamin E. Ann. Nutr. Metab. 39:1-8.

19. Ylä-Herttuala, S. 1991. Macrophages and oxidized low density lipoproteins in the pathogenesis of atherosclerosis. Ann. Med. 23:561-567.

20. Henriksen, T., E.M. Mahoney, and D. Steinberg. 1983. Enhanced macrophage degradation of biologically modified low density lipoprotein. Arteriosclerosis. 3:149-159.

21. Quinn, M.T., S. Parthasarathy, L.G. Fong, and D. Steinberg. 1987. Oxidatively modified low density lipoproteins: a potential role in recruitment and retention of monocyte/macrophages during atherogenesis. Proc. Natl. Acad. Sci. USA. 84:2995-2998.

22. McMurray, H.F., S. Parthasarathy, and D. Steinberg. 1993. Oxidatively modified low density lipoprotein is a chemoattractant for human $\mathrm{T}$ lymphocytes. J. Clin. Invest. 92:1004-1008.

23. Autio, I., O. Jaakkola, T. Solakivi, and T. Nikkari. 1990. Oxidized lowdensity lipoprotein is chemotactic for arterial smooth muscle cells in culture. FEBS Lett. 277:247-249.

24. Hessler, J.R., D.W. Morel, L.J. Lewis, and G.M. Chisolm. 1983. Lipoprotein oxidation and lipoprotein-induced cytotoxicity. Arteriosclerosis. 3:215222

25. Parthasarathy, S., D. Steinberg, and J.L. Witztum. 1992. The role of oxidized low-density lipoproteins in the pathogenesis of atherosclerosis. Annu. Rev. Med. 43:219-225.

26. Haberland, M.E., and U.P. Steinbrecher. 1992. Modified low-density lipoproteins: Diversity and biological relevance in atherogenesis. Monogr. Hum. Genet. 14:35-61.

27. Addis, P.B., H.A. Emanuel, S.D. Bergmann, and J.H. Zavoral. 1989. Capillary GC quantification of cholesterol oxidation products in plasma lipoproteins of fasted humans. Free Radic. Biol. Med. 7:179-182.

28. Kita, T., Y. Nagano, M. Yokode, K. Ishii, N. Kume, A. Ooshima, H Yoshida, and C. Kawai. 1987. Probucol prevents the progression of atherosclerosis in Watanabe heritable hyperlipidemic rabbit, an animal model for familial hypercholesterolemia. Proc. Natl. Acad. Sci. USA. 84:5928-5931.

29. Gaziano, J.M., J.E. Manson, J.E. Buring, and C.H. Hennekens. 1992. Dietary antioxidants and cardiovascular disease. Ann. N. Y. Acad. Sci. USA. 669:249-258.

30. Chisolm, G.M., G. Ma, K.C. Irwin, L.L. Martin, K.G. Gunderson, L.F. Linberg, D.W. Morel, and P.E. DiCorleto. 1994. 7 $\beta$-hydroperoxycholest-5-en$3 \beta$-ol, a component of human atherosclerotic lesions, is the primary cytotoxin of oxidized human low density lipoprotein. Proc. Natl. Acad. Sci. USA. 91: $11452-11456$.

31. Natarajan, V., W.M. Scribner, and M.M. Taher. 1993. 4-Hydroxynonenal, a metabolite of lipid peroxidation, activates phospholipase D in vascular endothelial cells. Free Radic. Biol. Med. 15:365-375.

32. DiCorleto, P.E., and C.A. De la Motte. 1985. Characterization of the adhesion of the human monocytic cell line U937 to cultured endothelial cells. $J$. Clin. Invest. 75:1153-1161.

33. Fox, P.L., and P.E. DiCorleto. 1986. Modified low density lipoproteins suppress production of a platelet-derived growth factor-like protein by cultured endothelial cells. Proc. Natl. Acad. Sci. USA. 83:4774-4778.

34. Lowry, O.H., N.J. Rosebrough, A.L. Farr, and R.J. Randall. 1951. Protein measurement with the Folin phenol reagent. J. Biol. Chem. 193:265-275.

35. Schuh, J., G.F. Fairclough, and R.H. Haschemeyer. 1978. Oxygen-mediated heterogeneity of apo-low-density lipoprotein. Proc. Natl. Acad. Sci. USA. 75:3173-3177.

36. Sparrow, C.P., S. Parthasarathy, and D. Steinberg. 1988. Enzymatic modification of low density lipoprotein by purified lipoxygenase plus phospholipase $\mathrm{A}_{2}$ mimics cell-mediated oxidative modification. J. Lipid Res. 29:745-753.

37. Jougasaki, M., K. Kugiyama, Y. Saito, K. Nakao, H. Imura, and H. Yasue. 1992. Suppression of endothelin-1 secretion by lysophosphatidylcholine in oxidized low density lipoprotein in cultured vascular endothelial cells. Circ.
Res. 71:614-619.

38. Geurts van Kessel, W.S.M., W.MA. Hax, R.A. Demel, and J. de Gier. 1977. High performance liquid chromatographic separation and direct ultraviolet detection of phospholipids. Biochim. Biophys. Acta. 486:524-530.

39. Nakamura, K., and S. Handa. 1984. Coomassie brilliant blue staining of lipids on thin-layer plates. Anal. Biochem. 142:406-410.

40. Kume, N., M.I. Cybulsky, and M.A. Gimbrone, Jr. 1992. Lysophosphatidylcholine, a component of atherogenic lipoproteins, induces mononuclear leukocyte adhesion molecules in cultured human and rabbit arterial endothelial cells. J. Clin. Invest. 90:1138-1144.

41. Schmidt, K., P. Klatt, W.F. Graier, G.M. Kostner, and W.R. Kukovetz 1992. High-density lipoprotein antagonizes the inhibitory effects of oxidized low-density lipoprotein and lysolecithin on soluble guanylyl cyclase. Biochem. Biophys. Res. Commun. 182:302-308.

42. Hamilton, T.A., G.P. Ma, and G.M. Chisolm. 1990. Oxidized low density lipoprotein suppresses the expression of tumor necrosis factor- $\alpha$ mRNA in stimulated murine peritoneal macrophages. J. Immunol. 144:2343-2350.

43. Berliner, J.A., M.C. Territo, A. Sevanian, S. Ramin, J.A. Kim, B. Bamshad, M. Esterson, and A.M. Fogelman. 1990. Minimally modified low density lipoprotein stimulates monocyte endothelial interactions. J. Clin. Invest. 85: 1260-1266.

44. Fong, L.G., T.A.T. Fong, and A.D. Cooper. 1991. Inhibition of lipopolysaccharide-induced interleukin- $1 \beta$ mRNA expression in mouse macrophages by oxidized low density lipoprotein. J. Lipid Res. 32:1899-1910.

45. Parthasarathy, S., and J. Barnett. 1990. Phospholipase $A_{2}$ activity of low density lipoprotein: evidence for an intrinsic phospholipase $\mathrm{A}_{2}$ activity of apoprotein B-100. Proc. Natl. Acad. Sci. USA. 87:9741-9745.

46. Quinn, M.T., S. Parthasarathy, and D. Steinberg. 1988. Lysophosphatidylcholine: A chemotactic factor for human monocytes and its potential role in atherogenesis. Proc. Natl. Acad. Sci. USA. 85:2805-2809.

47. Kugiyama, K., S.A. Kerns, J.D. Morrisett, R. Roberts, and P.D. Henry. 1990. Impairment of endothelium-dependent arterial relaxation by lysolecithin in modified low-density lipoproteins. Nature (Lond.). 344:160-162.

48. Kume, N., and M.A. Gimbrone. 1994. Lysophosphatidylcholine transcriptionally induces growth factor gene expression in cultured human vascular endothelial cells. J. Clin. Invest. 93:907-911.

49. Chatterjee, S. 1992. Role of oxidized human plasma low density lipoproteins in atherosclerosis: effects on smooth muscle cell proliferation. Mol. Cell. Biochem. 111:143-147.

50. Sakai, M., A. Miyazaki, H. Hakamata, T. Sasaki, S. Yui, M. Yamazaki, M. Shichiri, and S. Horiuchi. 1994. Lysophosphatidylcholine plays an essential role in the mitogenic effect of oxidized low density lipoprotein on murine macrophages. J. Biol. Chem. 269:31430-31435.

51. Hoffman, R.D., M. Kligerman, T.M. Sundt, N.D. Anderson, and H.S Shin. 1982. Stereospecific chemoattraction of lymphoblastic cells by gradients of lysophosphatidylcholine. Proc. Natl. Acad. Sci. USA. 79:3285-3289.

52. Tokumura, A., M.H. Mostafa, D.R. Nelson, and D.J. Hanahan. 1985. Stimulation of $\left(\mathrm{Ca}^{2+}+\mathrm{Mg}^{2+}\right)$-ATPase activity in human erythrocyte membranes by synthetic lysophosphatidic acids and lysophosphatidylcholines. Effects of chain length and degree of unsaturation of the fatty acid groups. Biochim. Biophys. Acta. 812:568-574.

53. Lundbæk, J.A., and O.S. Andersen. 1994. Lysophospholipids modulate channel function by altering the mechanical properties of lipid bilayers. J. Gen. Physiol. 104:645-673.

54. Jalink, K., W.H. Moolenaar, and B. Van Duijn. 1993. Lysophosphatidic acid is a chemoattractant for Dictyostelium discoideum amoebae. Proc. Natl Acad. Sci. USA. 90:1857-1861.

55. Weltzien, H.U. 1979. Cytolytic and membrane-perturbing properties of lysophosphatidylcholine. Biochim. Biophys. Acta 559:259-287.

56. Sugiyama, S., K. Kugiyama, M. Ohgushi, K. Fujimoto, and H. Yasue. 1994. Lysophosphatidylcholine in oxidized low-density lipoprotein increases endothelial susceptibility to polymorphonuclear leukocyte-induced endothelial dysfunction in porcine coronary arteries: role of protein kinase C. Circ. Res. 74 $565-575$.

57. Kugiyama, K., M. Ohgushi, S. Sugiyama, T. Murohara, K. Fukunaga, E. Miyamoto, and H. Yasue. 1992. Lysophosphatidylcholine inhibits surface receptor-mediated intracellular signals in endothelial cells by a pathway involving protein kinase C activation. Circ. Res. 71:1422-1428.

58. Flavahan, N.A. 1993. Lysophosphatidylcholine modifies G proteindependent signaling in porcine endothelial cells. Am. J. Physiol. 264:H722H727.

59. Sa, G., and P.L. Fox. 1994. Basic fibroblast growth factor-stimulated endothelial cell movement is mediated by a pertussis toxin-sensitive pathway regulating phospholipase $\mathrm{A}_{2}$ activity. J. Biol. Chem. 269:3219-3225.

60. Switzer, S., and H.A. Eder. 1965. Transport of lysolecithin by albumin in human and rat plasma. J. Lipid Res. 6:506-511.

61. Portman, O.W., and D.R. Illingworth. 1973. Lysolecithin binding to human and squirrel monkey plasma and tissue components. Biochim. Biophys. Acta. 326:34-42.

62. Portman, O.W., and M. Alexander. 1969. Lysophosphatidylcholine concentration and metabolism in aortic intima plus inner media: effect of nutritionally induced atherosclerosis. J. Lipid Res. 10:158-165. 
63. Portman, O.W., and D.R. Illingworth. 1974. Metabolism of lysolecithin in vivo and in vitro with particular emphasis on the arterial wall. Biochim. Biophys. Acta. 348:136-144.

64. Subbaiah, P.V., C. -H. Chen, J.D. Bagdade, and J.J. Albers. 1985. Substrate specificity of plasma lysolecithin acyltransferase and the molecular species of lecithin formed by the reaction. J. Biol. Chem. 260:5308-5314.

65. Subbaiah, P.V., and H. Monshizadegan. 1988. Substrate specificity of human lecithin-cholesterol acyltransferase towards molecular species of phosphatidylcholine in native plasma. Biochim. Biophys. Acta 963:445-455.

66. Reidy, M.A., A.W. Clowes, and S.M. Schwartz. 1983. Endothelial regeneration. V. Inhibition of endothelial regrowth in arteries of rat and rabbit. Lab. Invest. 49:569-575.

67. Minick, C.R., M.B. Stemerman, and W. Insull. 1977. Effect of regenerated endothelium on lipid accumulation in the arterial wall. Proc. Natl. Acad.
Sci. USA. 74:1724-1728

68. Walker, L.N., and D.E. Bowyer. 1984. Endothelial healing in the rabbit aorta and the effect of risk factors for atherosclerosis. Hypercholesterolemia Arteriosclerosis. 4:479-488.

69. Morel, D.W., P.E. DiCorleto, and G.M. Chisolm. 1984. Endothelial and smooth muscle cells alter low density lipoprotein in vitro by free radical oxidation. Arteriosclerosis. 4:357-364.

70. Steinbrecher, U.P., S. Parthasarathy, D.S. Leake, J.L. Witztum, and D. Steinberg. 1984. Modification of low density lipoprotein by endothelial cells involves lipid peroxidation and degradation of low density lipoprotein phospholipids. Proc. Natl. Acad. Sci. USA. 81:3883-3887.

71. Kalant, N., S. McCormick, and M.A. Parniak. 1991. Effects of copper and histidine on oxidative modification of low density lipoprotein and its subsequent binding to collagen. Arterioscler. Thromb. 11:1322-1329. 